\title{
Study on the Preparation and Influence Factors of Wood Assembled Adhesive in Normal Pressure
}

\author{
Qing Xiang, Xiaoyue Liu, Chao Zhu, Liming Dong* \\ School of Chemical Engineering, Xuzhou Institute of Technology, Xuzhou, 221111, China
}

*Corresponding author: Liming Dong

\begin{abstract}
To solve the problem of the strong adhesive in normal temperature and pressure, a new wood assembled adhesive based on water-based polymer - isocyanate adhesive (API) is developed. The effect of prescription composition, viscosity, solid content and cross-link agent content were studied by laser particle size analyzer and mechanical property test on the properties of adhesive. when the content of polyvinyl acetate emulsion $(P V A C)$, vinyl acetate - ethylene co-polymer emulsion (VAE), polyvinyl alcohol(PVA), heavy calcium were weight ratio of $37.5 \%, 25 \%, 6 \%, 2.5 \%$, and the polyaryl methene isocyanate $(P A P I)=8 \%$ (hostbased quality), the results show that wood assembled adhesive exhibits excellent adhesive strength(13.6 $M P a)$.
\end{abstract}

KEYWORD: Water-based polymer-isocyanate; wood assembled adhesive; emulsion

\section{INTRODUCTION}

With the rise of modem furniture design, the components assembled mode more and more replaced the traditional furniture manufacture mode (K. Xue, 2013). Common methods of assembling usually include cogged joint, nail joint, Connector joint and adhesive and etc. Adhesive technology is applied more and more extensively in the wood furniture manufacturing (X.CH. Yu et al, 2004) (J.Y. Gu, 2006) because of better process, simple equipment, easy operation, low cost, wide application, good sealing corrosion resistance, high strength of resistance fatigue. In addition, Adhesive technology is applied widely in the cogged joint, overlaying process, plate alignment and etc. With people's strengthened environmental awareness and robust regulations about national environmental protection, the problems about pollution in wood furniture adhesive are getting people's attention.

In 1980s, Japan first developed a water-based polymer - isocyanate adhesive (API) formed by water-soluble polymers, polymer emulsion, fillers as based agent and polyfunctional isocyanate compound as cross-linking agent (J.Y. Gu et al, 2005). Compared with urea formaldehyde resin (UF), melamine - formaldehyde resin $(M F)$, phenol resin $(P F)$, $A P I$ is an environmentally adhesive owning noformaldehyde and using conveniently and safely. Outstanding advantages of API include cured at room temperature, water resistance, excellent heat resistance and anti-aging properties, while reasonable price, and widely used in the production of small cross section construction timber, furniture puzzle, doors and windows, plywood, decorative board widely. Its market can be broken down as follows: integrated board adhesive occupied 45\%; puzzle furniture occupied 30\%; plywood (including formaldehyde-free plywood and parquet core) occupied $15 \%$; other (veneer sheets, metal foil, plastic sheet wood and composite with wood-based panels) accounted for $10 \%$ (L.J. Chen et al, 2001).

In order to obtain the target adhesive strength and effectiveness, API adhesives often use hot-pressing process. Higher temperature accelerated isocyanate group of curing agent to react with the primary agent or wood surface active groups, thus contributing to produce more chemical cross-linking structure to improve the adhesive strength. Higher pressure accelerated adhesive infiltration to the surface of the wood cell lumen, thus contributing to produce more the contact area of the combined interface; Higher pressure accelerated adhesive colloidal particles diffusion and fusion, thus contributing to produce more continues film between wood and wood surface sufficiently to achieve bonding; Higher pressure accelerated the elimination of $\mathrm{CO}_{2}$ gases due to the isocyanate-reactive, thus contributing to reduce more air bubble defect remaining in the film. In comparison, the change of pressure was more conducive to improving properties of adhesive (H.Y. Liu et al, 2013) (J. Zhang et al, 2009) (He Lie, 2009). 
In regard of the production of timer and plate, the pressing operation is better convenient, and common API adhesive can achieve reliable bonding. However, the poor conditions of pressure value and direction exist widely in the actual furniture assembly production process (K.D. Gao, 2009) (Zh.X. Zhou et al, 2008). The adhesive must have higher performance that the strength of joints under low pressure can reach more than the strength of assembly components themselves.

\section{EXPERIMENTAL}

\subsection{Materials.}

PVA(BP-24, BP-17)was obtained from Taiwan Changchun Co., Ltd.; PVAc emulsion (502) was obtained from Anhui Wanwei Co., Ltd., solid content $50 \%$; VAE emulsion (CP149) was obtained from Celanese Co., Ltd., solid content 50\%; Vinyl acetate - ethylene co-polymer emulsion (VAE), 50\% solids CP149 emulsion, was purchased from International Trade Corporation Limited; PAPI(PM-400) as crosslink agents was obtained from Yantai Wanhua Polyurethane Co., Ltd.; Film-forming additive (DBP, CS12 and DPM), Dispersing agent (SN-5040), Leveling agent (BYK-333), Wetting agent (X-405), Deformers (NXZ), Mildew (CN46), heavy calcium and light calcium were gifted by the QiYanli paint Co., Ltd.

\subsection{Preparation and Test.}

A suitable amount of water is put into reaction vessel, and added PVA, inorganic filler, dispersing agent in proportion and feeding mode, then stirred and dispersed until the PVA is dissolved completely at $80-95^{\circ} \mathrm{C}$. The dispersion solution was added PVAC emulsion, VAE emulsion, pH buffers, Defoamer, wetting agents, flow agents, preservatives and stirred uniformly at $40-60^{\circ} \mathrm{C}$. The properties of main agent, $\mathrm{pH}=6.5-7.5$, viscosity of 15000-30000 MPa.s. The assembly adhesives can be obtain after cross-link agent is mixed with the main stirred uniformly. Compression shear strength was tested referencing the standard of HG/T 2727-2010, using specifications for $30 \mathrm{~mm} \times 25 \mathrm{~mm} \times 10 \mathrm{~mm}$ as birch specimens.

\section{RESULTS AND DISCUSSION THE FORMULATIONS OF MAIN AGENT}

\subsection{The Effects of VAE Emulsion Content on Adhesive Properties.}

The effects of VAE emulsion content on adhesive properties was shown in Fig. 1. The results show that the shear strength presents the tendency of increase first and reduction afterwards with the increase of VAE emulsion content. When the content of $V A E$ was $40 \%$, shear strength of the adhesive reached maximum. As shown in Fig. 2, the results of distribution for VAE and PVAc emulsion which were tested by laser particle size analyzer. The particle size of VAE emulsion was between 0.3 and $10 \mu \mathrm{m}$ and the biggest volume distribution was $1.79 \mu \mathrm{m}$; however the size of PVAC emulsion particle was between 50 and $500 \mu \mathrm{m}$ and the biggest volume distribution was $236.24 \mu \mathrm{m}$. It is obvious that the size of $V A E$ particle is smaller than that of PVAc for about two levels, so the permeability of VAE emulsion is better under the low pressure. It should be easier to fill into the gap in the wood fiber capillary and then strengthen the adhesive strength. Furthermore, VAE emulsion was co-polymer of ethylene and vinyl acetate monomer. Because the polymer backbone contains some parts of the ethylene segment, which played a role within the plasticized, increasing the flexibility of macro-molecule main chain, decreasing the glass transition temperature, improving the first viscosity. When the VAE contents increased above $40 \%$, the adhesive post-drying layer was more and softer, the adhesive showed weaken cohesion, shear strength presented decreasing trend. Therefore, the optimal value of VAE emulsion components was $40 \%$.

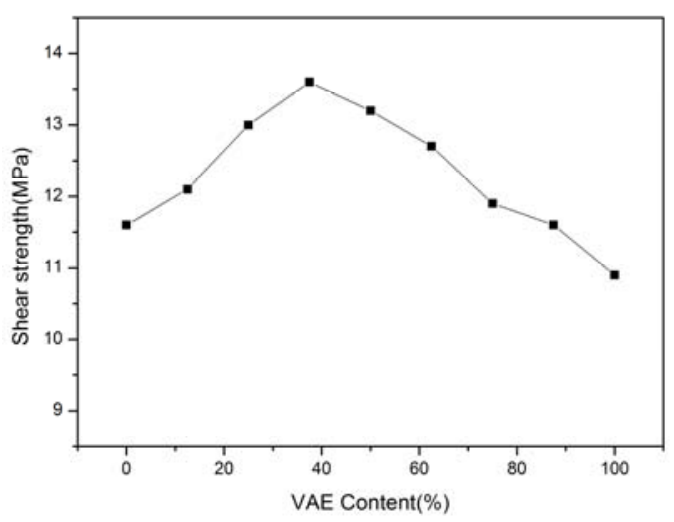

Fig. 1 The effects of VAE emulsion content on adhesive properties

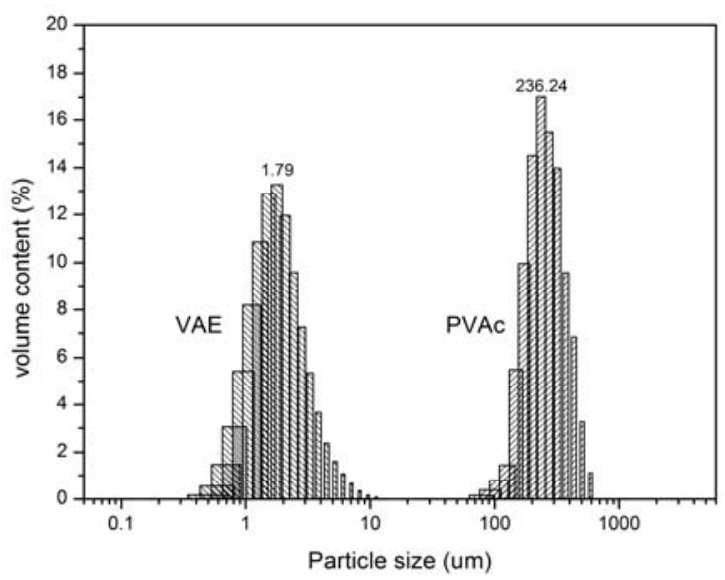

Fig. 2 The distribution results about VAE and PVAc emulsion particle size 


\subsection{The Impact of PVA Content on the Adhesive Properties.}

The impact of PVA content on the adhesive properties was shown in Fig. 3. To improve the effects of freeze-thaw resistance of adhesive, both alcoholysis degree of $P V A$ are all $88 \%$, the degree of polymerization of $B P-24(2400 \pm 50)$ is significantly higher than the $B P-17(1700 \pm 50)$. The results show that the shear strength presents the tendency of increase first and reduction afterwards with the increase of $P V A$ contents. When the content of $B P-24$ was $5 \%$, shear strength of the adhesive reached maximum12.5MPa. When the content of $B P-17$ was $6 \%$, shear strength of the adhesive reached maximum13.6MPa. It is found that the curing crosslinked reaction between hydroxyl groups on the PVA molecule chains with an isocyanate group quickly at room temperature, and the performance of the shear strength rises along with the increasing PVA content. However, with the continuous increase of PVA content, the curing speed was more quickly and the cross-link density was higher. It is more difficult for good shear strength and the defects of lack glue in adhesive layer were easy to take place. As a result of the viscosity of $B P-24$ is higher that it holds the same content and high polymerization degree, so the maximum shear strength occurs at lower levels. Nevertheless, BP-17 shown slow polymerization degree can improve the solid content of the active ingredient at low viscosity. Therefore, the amount of $B P-17$ is $6 \%$ selected preferably in the adhesive formulation.

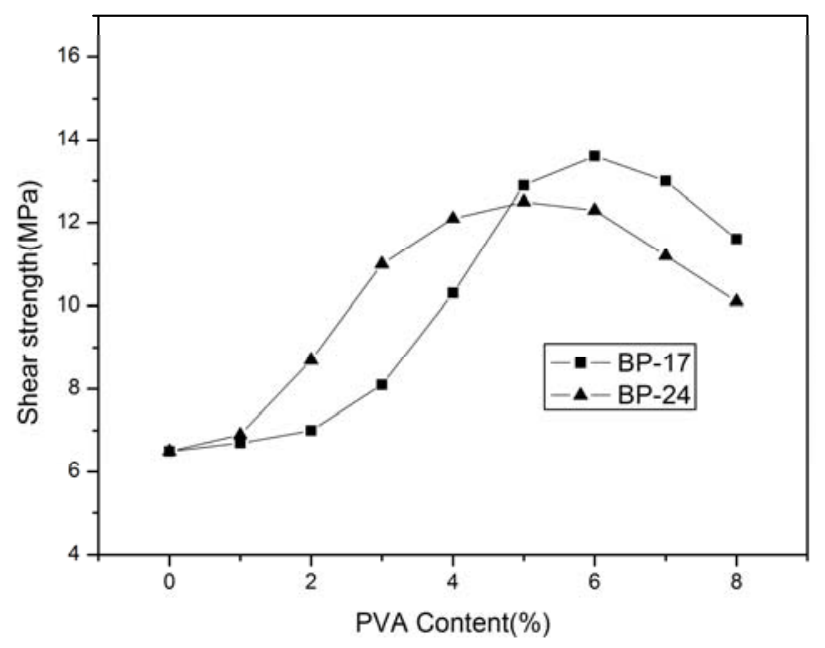

Fig. 3 The impact of PVA content on the adhesive properties

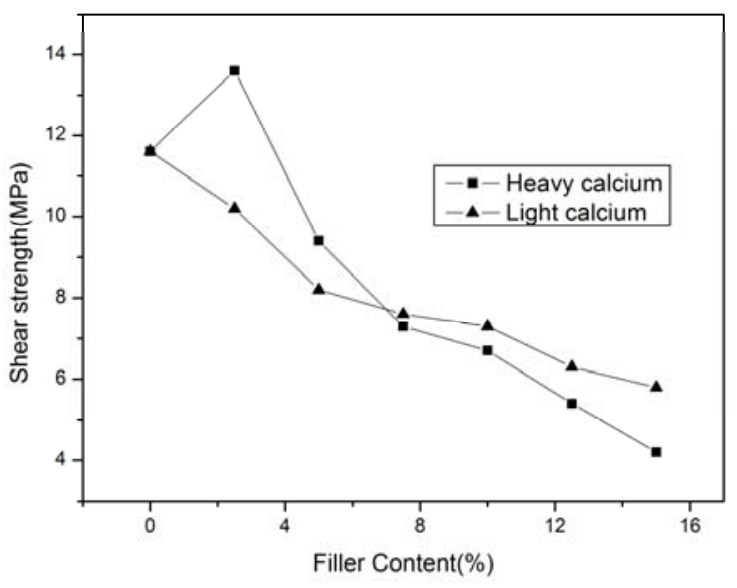

Fig. 4 The impact of the filler content on the adhesive properties

\subsection{The Impact of the Filler Content on the Adhesive Properties.}

The impact of the filler content on the adhesive properties was shown in Fig.4. The results show that the shear strength presents the tendency of reduction afterwards with the increase of light calcium. While heavy calcium appears sharp drop after the highest value $13.6 \mathrm{MPa}$ occurs at the content of $2.5 \%$. As is known to all that filler does some work to support load or absorb energy, meanwhile increase the impact resistance to some extent, so adhesive properties shows rising first and then declining owing to small amount of heavy calcium is added. However, the filler amount and particle size have an influence that the more amount of filler makes molecular movement difficulties and reduces the interface invasive on polymer molecular motion under stress so that shear strength decreases. Compared to light calcium, heavy calcium has a higher specific gravity, less amount of polymer adsorption and less impact on the connection effect. Therefore, a heavy calcium amount of $6 \%$ is selected as filler preferably in the adhesive formulation.

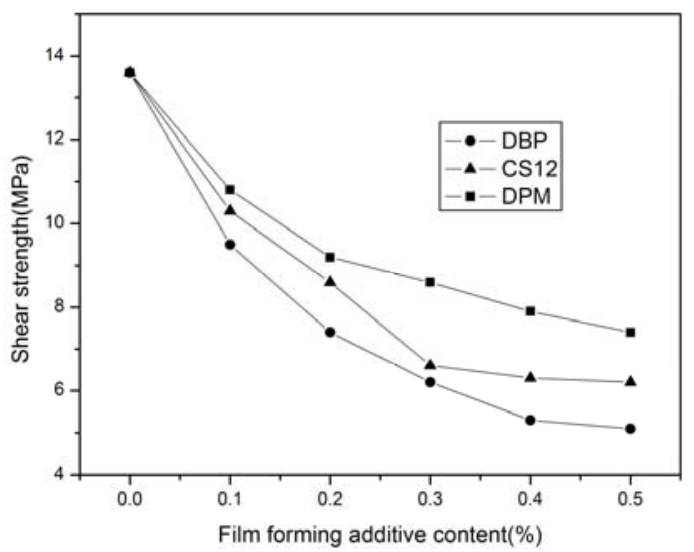

Fig. 5 The impact of film-forming additives content on the adhesive properties 


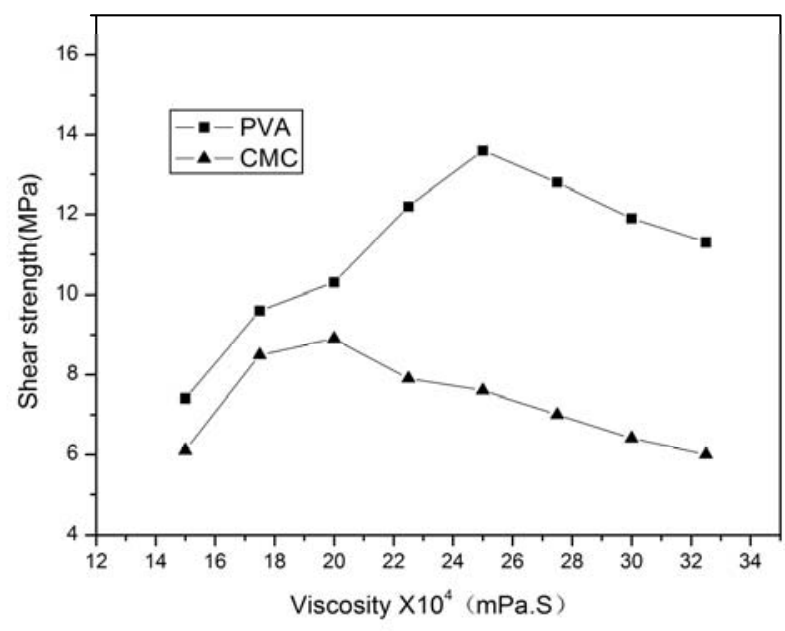

Fig. 6 The impact of Main Viscosity Index on the adhesive performance

\subsection{The Impact Content of Film-forming Additives on the Adhesive Properties.}

The impact of film-forming additives on the adhesive properties was shown in Fig. 5. The results show that the shear strength presents the tendency of reduction afterwards with the increase of film forming additive and the degree of decrease is $D B P>C S 12>D P M$. Due to the minimum film forming temperature of PVAC emulsion is high, the ability of polymer fusing to film-forming was weak which results adhesive force is poor and this situation often improved by adding plasticizer or filmforming additives. When PVAC emulsion and VAE emulsion mixed, VAE content have internally plasticized effect on whole adhesive. With the filmforming additives extra increase, volatile components in the adhesive was increasing, the shear strength decreased significantly. It is found that the boiling point of $D B P, C S 12$ and $D P M$ decreased, and the volatile increased, respectively. It was less the shear strength decreased in three film-forming additives because of volatility of $D P M$ is highest. Therefore, it is unnecessary to use film-forming additives in adhesive formulations.

From the analysis above, the main agent of adhesive proportion were : PVAc : VAE : PVA : heavy calcium $=37.5 \%: 25 \%: 6 \%: 2.5 \%$.Other proportions were lower than $1 \%$, such as $\mathrm{pH}$ buffering agent, defoamer, wetting agent, leveling agent, preservative. After 48 hours' curing under the normal temperature and pressure, the adhesive was tested by compressed shear strength method on universal mechanical testing machine. The result is up to $13.6 \mathrm{Mpa}$, and it reached the stander of $H G / T$ 2727-2010.

\subsection{The Impact of Main Viscosity on the Adhesive Performance.}

The practical invasive of adhesive is related to its viscosity or mobility, and the viscosity of main agent is related to its content. The influence of main agent viscosity on adhesive property is shown in Fig. 6 . The main agent viscosity can be adjusted by content and grades of PVA and CMC. The results show that the shear strength was increase first and then decrease with the viscosity increasing. For the reason of good mobility, the adhesive was easy to excessive glue and the effective constituent of unit surface was little, so the strength was poor. For the reason of bad mobility, the adhesive was difficult to infiltrate surface, so the strength was also poor. When viscosity was $25000 \mathrm{mPa}$.s in PVA viscosity curve, the highest value was $13.6 \mathrm{MPa}$. When viscosity was $20000 \mathrm{mPa}$.s in $C M C$ viscosity curve, the highest value was $8.7 \mathrm{MPa}$. Therefore, selecting $P V A$ to control the adhesive viscosity in formulations, and the best result appears when the viscosity is $25000 \mathrm{mPa}$.s.

\subsection{The Impact of the Main Agent Solid Content Index on the Adhesive Performance.}

The impact of the main agent solid content index on the adhesive performance is shown in Fig. 7. The results show that the shear strength presents the tendency of increase first and reduction afterwards with the increase of solid content. When the solid content was $40 \%$, shear strength of the adhesive reached maximum 13.6MPa. Both solids content of $V A E$ emulsion and PVAC emulsion are 50\%, which have little effect on the main agent. The solids content is affected mainly by the amount of PVA and filler. In initial stage, with the increase of solid content, the proportion of the polymer component adds, as well as effective adhesive components are more and the bonding effect increases significantly. In the late stage, the filler proportion increases while the components of effective adhesive and bonding effect decrease.

\subsection{The Impacts of Cross-linking Agent Content on the Adhesive Properties.}

The impacts on the adhesive properties of cross-link agent content is shown in Fig. 8, its content is the ratio of the cross-link agent and the main agent. The results show that the shear strength presents the tendency of increase first and reduction afterwards with the increase of cross-link agent content. Complete cross-linked network can not be formed as the content of cross-link agent is less, along with the less chemical reactions between cross-link agent and wood interface and the lower shear strength. With the cross-link agent increasing, the intensity is in- 
creased significantly. When the cross-link agent content was $8 \%$, shear strength of the adhesive reached maximum 13.6MPa. When excessive crosslinking agent was used, the viscosity of adhesives in its useful life will increase along with the weakened adhesive fluidity and infiltrating so as to decrease the strength. Contrary to the optimum amount of cross-link agent was $15 \%$ under the conditions of heating and pressurizing in the literature, cross-link agent content used in present formulation was less, which may be related to the slower cross-link reaction rate.

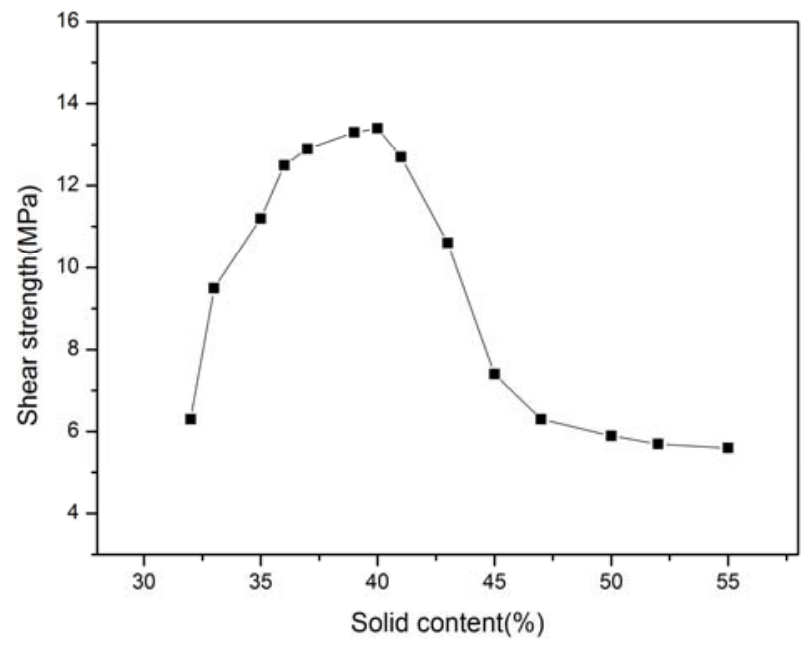

Fig. 7 The impact of the main agent solid content index on the adhesive performance

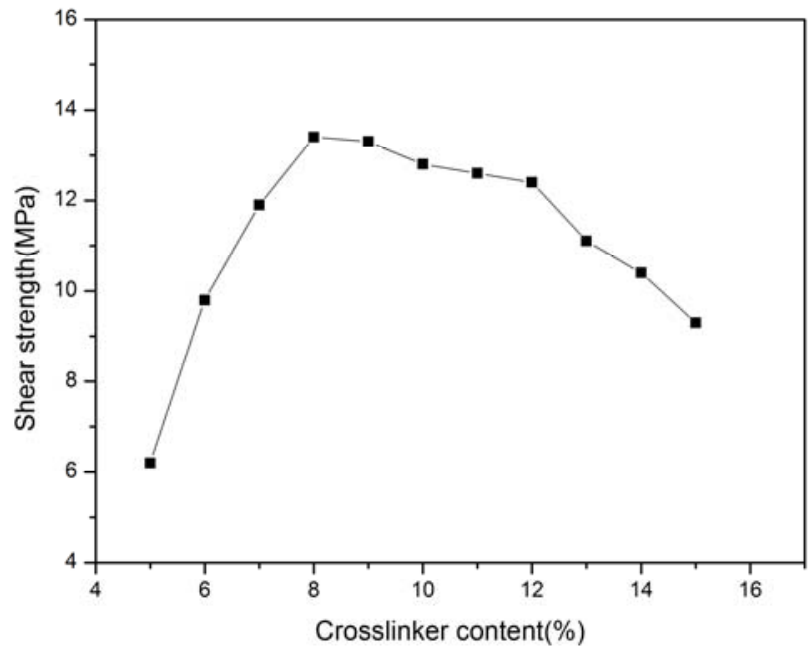

Fig. 8 The impacts of cross-linking agent content on the adhesive properties

\section{CONCLUSION}

A new wood assembled adhesive is developed for normal temperature and pressure application conditions, which were made up of PVAC and VAE emulsion as main components -primary agent and PAPI as cross-link agent. The performance of adhesive is optimal when the content that $P V A c: V A E: P V A$ :heavy calcium in a weight ratio of $37.5 \%: 25 \%: 6 \%: 2.5 \%, P A P I=8 \%$ (host-based quality).The adhesive is very suitable for manufacturing and assembly of wooden furniture that an ideal wood adhesive production, which owns excellent weather resistance and adhesive strength can be achieved 13.6 $\mathrm{MPa}$ by curing 48 hours under normal temperature and pressure.

\section{ACKNOWLEDGMENTS}

The project was supported by the Natural Science Foundation of Jiangsu Province (No. BK2012142)

\section{REFERENCES}

He Lie. Study of Normal Temperature Fast-solidify Emulsion Timber Adhesive, (Ph.D., Nanjing Forestry University, China 2009), p.25.

H.Y. Liu, H. Zhao, F.CH. Xiao, Y.H. Zhang and J.Y. Gu, J. Chemistry and Adhesion, 2013 (5): 1-4.

J.Y. Gu, G.R. Zhou, J. China Adhesives, 2005, 14(6): 29-30.

J.Y. Gu, J. China Wood Industry, 2006, 20(2): 66-68.

J. Zhang, J.Y. Gu, Y.H. Zhang, G.B. Zhu, J. China Adhesives, 2009, 18(2): 1-4.

K.D. Gao, J. Forestry Science and Technology, 2009 (3): 5355.

K. Xue, Analysis on the Structural Performance of Traditional Chinese Furniture and the Design Improvement of Mortise and Tenon Joint (Ph.D., Nanjing Forestry University, China 2013), p.1.

L.J. Chen, J.Y. Gu, J. Zhan Jie, 2001, 22(3): 9-10.

X.CH. Yu, Zh. Chen, D.L. Sun, J. China Forest Products industry, 2004, 3: 51-53.

Zh.X. Zhou, X.F. Li, J. Journal of Inner Mongolia Agricultural University, 2008, 29(1): 144-147. 